\title{
Development of a Unified and Dynamic Geometric Framework for Modelling Plant Leaf Spots
}

\author{
Duaa Alshadli \\ School of Construction and Civil \\ Engineering at Unitec Institute of \\ Technology \\ +64981543217601 \\ dalshadli@unitec.ac.nz
}

\author{
Kambiz Borna \\ School of Construction and Civil \\ Engineering at Unitec Institute of \\ Technology \\ +64981543217186 \\ kborna@unitec.ac.nz
}

\author{
Cesar Lador \\ School of Construction and Civil \\ Engineering at Unitec Institute of \\ Technology \\ $+64981543217421$ \\ clador@unitec.ac.nz
}

\begin{abstract}
The use of computer vision and image processing techniques have proven to be effective in detecting and classifying plant diseases from symptoms, such as leaf spots or mosaic leaf patterns caused by pathogens. To identify these symptoms, these techniques typically utilize a static geometry specified by a human expert via pixels or image objects. Thus the results rely on generic parameters defined by the user before or after classification. In this paper, a dynamic geometry is proposed that can be applied to identify plant disease symptoms without setting any geometric parameters. The offered method consists of two primary phases established based on the notion of Vector Agents (VAs): construction of a unified geometry, and creation of a dynamic geometry. In the construction step, the method utilises a set of geometric rules to link the raster space to the vector space during the simulation process. These rules specify the growth direction of a leaf in the simulation space and the maximum length of each edge specified based on the image spatial resolution and size. The creation step includes four main geometric operators: 1) vertex displacement, 2) half-edge joining, 3 ) converging vertex displacement, and 4) edge remove. This enables the leaves to automatically change their geometry in the simulation space without setting any geometric parameters. This structure allows a classifier to model a wide range of leaf shapes in the image space. The proposed geometry method was tested to model different leaves and the results demonstrate its effectiveness in generating different leaf shapes.
\end{abstract}

\section{CCS Concepts}

Applied computing $\rightarrow$ Computers in other domains $\rightarrow$ Agriculture.

\section{Keywords}

Dynamic geometry, Image object, Plant disease, leaf spots, Vector agents.

\section{INTRODUCTION}

Plant diseases are a major cause of agricultural loss, accounting for more than $20 \%$ of annual loss and poses a continuous threat to agricultural production [1]. To minimise this effect, numerous diagnostic techniques, e.g., remote sensing imagery, have been developed to target and diagnose diseases. These techniques usually utilise computer vision and image processing algorithms to identify visible disease symptoms such as leaf spots [2]- [4].

The identification process is typically performed based on image classification, which is the process of labelling pixels or image objects. For pixel-based classification, the spectral information of each pixel is applied to label the pixels in a feature space via a machine learning algorithm such as the Mahalanobis algorithm [3]. The classified images are then used to identify unhealthy leaves while limiting the algorithm from utilising the spatial information of leaves, e.g. geometry, particularly at the leaf level. To address this issue, advanced pixel-based classification methods can be utilised to apply an additional image processing technique such as the Canny edge detection method [3], [5]. These methods use the gradient amplitude between pixels in a local neighborhood window to identify the leaf edge pixels. Despite the advantages of these algorithms, particularly around leaf boundary pixels, they lack the ability to use the areal information of leaves for the identification of leaf diseases. Moreover, they are sensitive to noise and can easily detect false leaf edges and lose real edges especially when high spatial resolution images are used.

To deal with this limitation, image classification algorithms employ a segmentation process to create image objects (a set of connected pixels). These objects allow the algorithms to use more spatial information of leaves, e.g. geometry and texture, compared to the advanced pixel-based methods. For example, [2] used a genetic algorithm where they introduced texture features such as homogeneity and contrast to segment an image. The segmented image was then applied by a Support Vector Machine (SVM) algorithm to identify leaf spots. Reference [4] proposed a weighted segmentation method, formulated based on the Chi-square distance and threshold function, to extract citrus lesion spots. The information of the segmented objects, e.g., area, perimeter, and texture, was then applied by the SVM algorithm to classify citrus diseases. To take advantage of the spatial information, [6] applied a combination of strongly correlated pixels and an expectation maximization algorithm in order to identify the initial locations of apple leaf spots. They also employed the SVM algorithm to classify extracted objects in the segmentation step. These techniques have two characteristics in common; they use a sequential process of image segmentation and image classification to detect a leaf disease, and they apply a static geometry based on parameters specified by a human expert to identify leaf spots. However, these methods do not have the capability of offering a unique solution to determine the optimum values of these parameters. In other words, similar to the advanced pixel-based approaches, these methods lack the necessary abilities to implement a unified and dynamic geometry to simultaneously address interior and exterior boundaries of leaves.

Through this paper, a new approach is proposed that allows a classifier to model the interior and exterior boundaries of leaves without setting any user-defined parameters. The method is established on a unified and dynamic geometry, which enables a classifier algorithm such as SVM to simultaneously model a leaf object in the same way that a human interpreter does from an image without setting any additional geometric parameters. 


\section{METHOD}

\subsection{Plant Disease Symptoms}

Plant diseases caused by pathogens typically exhibit visible symptoms such as changes in colour, texture or shape of parts of plants. Figure 1(a) and (b) display the effects of fungal pathogens on plant leaves, such as rust spots with irregular shapes (Figure 1(a)) and angular leaf spots near veins with circular shapes (Figure 1(b)).

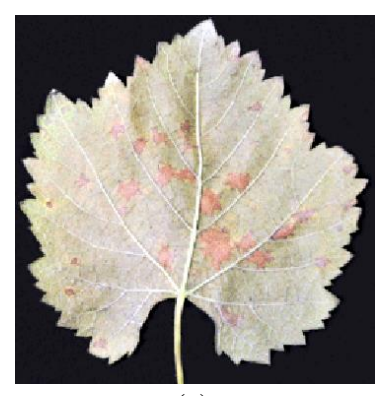

(a)

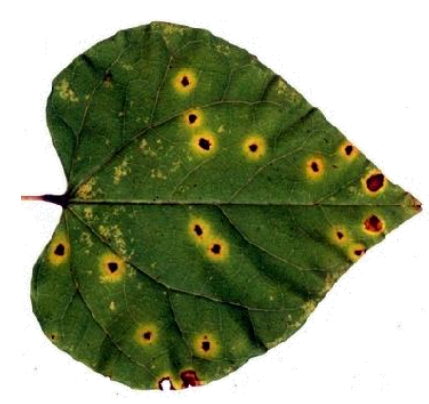

(b)
Figure 1. Leaf spot caused by Neopestalotiopsis vitia (a) [7]. Leaf spot on common persimmon caused by Pseudocercospora vitis (b) [8].

As disease spots have different shapes, it is necessary for an applied method to have the ability to model a wide range of geometries. This can be performed through a segmentation algorithm that addresses the exterior and interior structure of leaves separately through a static geometry. We propose a unified and dynamic geometry to enable a classifier to simultaneously model interior and exterior leaf boundaries. The method is implemented in two main steps: 1) the construction of a unified geometry, and 2) the creation of a dynamic geometry. To create the model, we used the notion of the Vector Agents (VAs) proposed by [9] and [10]. According to [9], at a primary level, VAs can be considered as a set of objects that dynamically change their own geometry in a simulation space. The algorithm was implemented using Repast, a Java-based Toolkit on an Intel CPU at $1.70 \mathrm{GHz}$ and $4 \mathrm{~GB}$ of memory. ArcGIS v.10.5 and eCognition v.9 software were also used to analyse the images and to represent the $2 \mathrm{D}$ view of the generated maps.

\subsection{Construction of the Unified Geometry}

In the proposed method, the simulation space is a vector space with polygons that together represent a leaf within an image. Let $x=\left(x_{1}, x_{2}, x_{3}, \cdots, x_{n}\right) \in \mathbb{R}^{d}$ denote a multispectral $d$-dimensional image with $n$ pixels, $l=$ $\left(l_{1}, l_{2}, l_{3}, \cdots, l_{m}\right) \in \mathbf{L}$ include a set of coordinates where, $m<n$ and $l_{m}=\left(x_{\mathrm{m}}, y_{\mathrm{m}}\right)$. With this notation, $\mathbf{L}$ stores the vertices of the polygon that define the boundary of the leaf, and uses an adjacency list algorithm to store the coordinates of each vertex In this case, for each vertex $v$ in the polygon (leaf object), $\mathbf{L}$ is defined via all points of $v$ to $v$. The geometry and topology of faces, edges, and vertices of the graph are formulated based on the winged-edge data structure [11]. This allows each edge $e$ within the polygon to have two faces incident to $e$, e.g. edge ab in Figure 2(c), which enables the method to efficiently model the interior and exterior boundaries of a leaf. The method applies the rules defined by [12] and [13] to link the polygons in the simulation (vector) to the image (raster) space:

- $l_{m}\left(x_{\mathrm{m}}, y_{\mathrm{m}}\right)$ is defined based on the coordinates of the pixel centre;

- $\quad$ each vertex can generate a new vertex along four cardinal directions at a set distance corresponding to the raster cell size $\mathrm{r}$ (Figure 2(a)), which can support a twin-edge (e.g. ab in Figure 2(b)),

- each new vertex has three reference points,

- $\quad$ an edge can be created if there is a vertex in the local neighbourhood (eight cardinal directions) of the new vertex (Figure 2(b), (c) and (d)), and

- each edge has a maximum length of $r \sqrt{2}$ (the diagonal distance of a pixel). For example, in Figure 2(b), the method cannot create edge bd1.

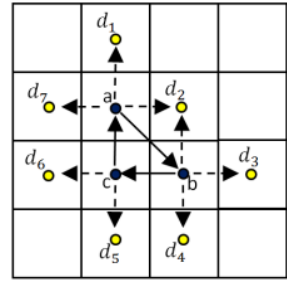

(a)

$$
\begin{aligned}
& \text { - Reference point } \\
& \text { - Possible new point } \\
& \rightarrow \text { Possible new edge }
\end{aligned}
$$
$\rightarrow$ Main edge

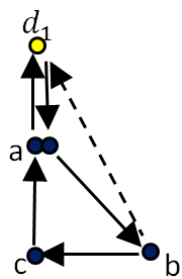

(b)

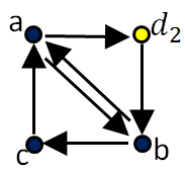

(c)

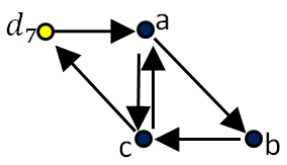

(d)
Figure 2. (a) displays a three sided polygon $L(a, b, c)$ from a vector space superimposed on the raster image at time $t$. (b),

(c) and (d) display three different shapes of a four sided polygon based on vertex at time $t+1$.

The following equation was applied to find the orientation of each face (interior or exterior boundaries) in the winged-edge data structure:

$$
\left[\begin{array}{lll}
i & j & k
\end{array}\right]=\operatorname{sgn}\left|\begin{array}{ccc}
x_{i} & x_{j} & x_{k} \\
y_{i} & y_{j} & y_{k} \\
1 & 1 & 1
\end{array}\right|
$$

where $\left(x_{\mathrm{i}}, y_{\mathrm{i}}\right)$ is the coordinate of each vertex and a positive value indicates a counterclockwise direction. These vertices form the geometry of the leaf object in the simulation space, and this structure allows the method to display a unified polygon $\mathbf{L}$ based on the pixels belonging to the leaf object in the image space.

\subsection{Creation of the Dynamic Geometry}

To enable the dynamic geometry of the leaf objects, a set of operators were applied as follows:

1. Vertex displacement: this places a new vertex in the space image. Figure 2(b) shows how the algorithm uses the geometric operator to create the twin-edge ad.

2. Half-edge joining: this constructs a new edge based on a twin edge that is formed by two half-edges (Figure 2(c)). 
3. Converging vertex displacement: two new edges are constructed to a single new neighbouring vertex from two existing vertices (Figure 2(d)).

4. Edge remove: this forms a new polygon by the merging of two polygons (Figure 3).

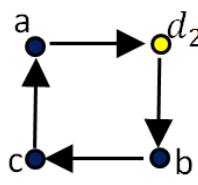

(a)

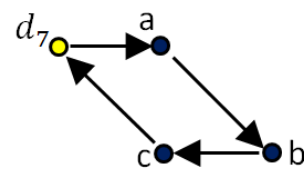

(b)
- Reference poin - Possible new point $\longrightarrow$ Main edge
Figure 3. (a) and (b) are formed based on Figure 2(c) and Figure 2(d) in terms of the edge remove operator, respectively.

These methods allow a leaf object to constantly change its geometry via an iterative mechanism without applying any userdefined geometric parameters. For example, Figures. 2(a), 2(c) and 3(a) show different steps of an evolving process from a three sided to a four-sided polygon. The algorithm first uses the point a to create point $\mathbf{d}_{\mathbf{2}}$ (Figure 2 (a)). Thus, the first edge is randomly constructed by finding a second point in the local neighbourhood in space (Figure 2 (c)). The new created edge consists of two half edges. The above geometric methods are then applied as required. Finally, the edge remove operator is utilized to eliminate the twin edge within the polygon (Figure 3(a)).

\section{EXPERMENTAL RESULTS}

The proposed approach was tested using two different images with different leaf geometries ((Figs. 1(a) and 1(b)). Figure 1(a), is an RGB image with a size of $136 \times 136$ pixels and displays leaf spots caused by Neopestalotiopsis vitis. Figure 1(b) is an RGB image with a size of $205 \times 205$ and displays symptoms of Pseudocercospora vitis.

Figure 4 shows an example of the evolving process for a single point through the implementation of the above rules and methods. The proposed geometry was applied by the SVM classifier, trained based on three different classes (leaf, non-leaf, and leaf spot) to model the leaf in Figure 1(a). The unified and dynamic structure of the proposed geometry allowed the SVM method to simultaneously create the interior and exterior boundaries of the leaf (Figure 4).

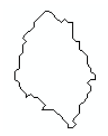

(a)

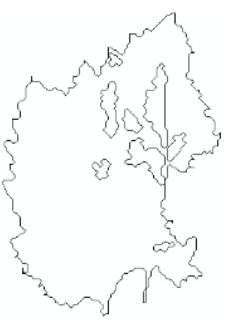

(c)

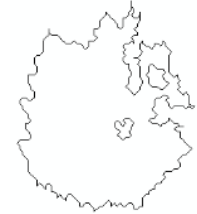

(b)

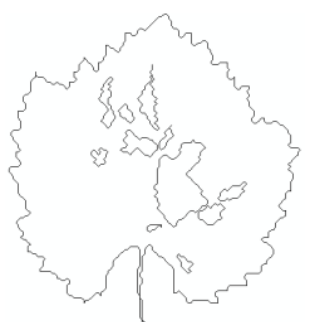

(d)
Figure 4. Simulation results in different epochs depicting the transformation from a point into a leaf object.
Figure 5 displays the extracted leaf objects via the proposed method superimposed on the images. The red polygons indicate the closeness of the generated leaf objects to the real structure of the leaves.

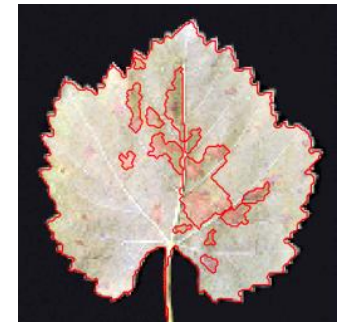

(a)

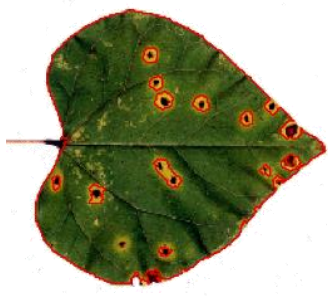

(b)
Figure 5. The extracted leaf objects from the proposed algorithm superimposed on the images in Figure 1.

To assess the performance of the proposed method, the extracted leaf objects were also compared with the results of the segmentation-based method using eCognition (Figure 6). Figure 6(b) shows that the segmented objects can readily determine the boundaries of leaf objects. However, the segmented objects cannot accurately address leaf objects where there is an indeterminate boundary and complex geometry (Figure 6 (a)). Because the segmentation-based method lacks the ability to change the geometry of the image objects in the classification step. Hence the geometry of the leaf object is different from the corresponding leaf in the image. Figure 6(b) shows that the segmented objects can readily determine the boundaries of leaf objects. However, the segmented objects cannot accurately address leaf objects where there is an indeterminate boundary and complex geometry (Figure 6 (a)). Because the segmentation-based method lacks the ability to change the geometry of the image objects in the classification step. Hence the geometry of the leaf object is different from the corresponding leaf in the image.

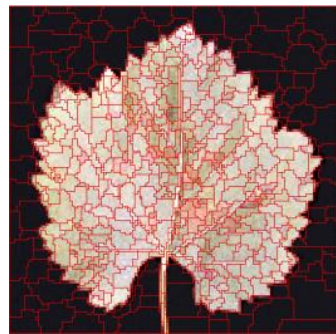

(a)

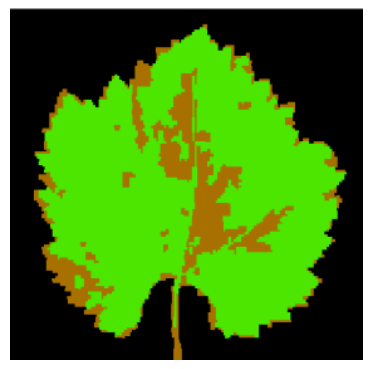

(c)

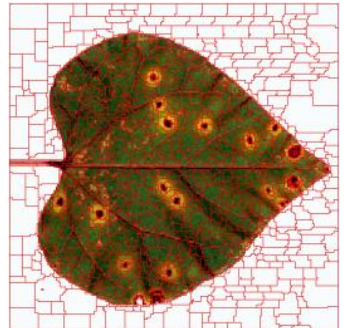

(b)

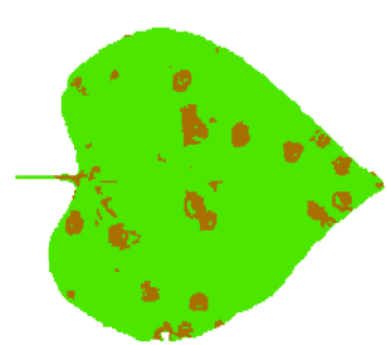

(d)
Figure 6. (a) and (b) display the segmented images created in eCognition. (c) and (d) are the classified images in terms of color and texture information of image objects. 
In order to make a quantitative assessment of the extracted leaf objects, the completeness or shape accuracy of the detected leaf objects (Table I) was measured by the following metric:

$$
\text { Shape }_{\text {accuracy }}=1-\frac{|A-B|}{A}
$$

where $A$ is the area of the leaf that is manually extracted (yellow polygons in Figure 7), and $B$ is the area extracted by the proposed method (blue polygons). To calculate the shape accuracy, the external leaf boundaries were considered. A visual assessment of Figure 7 shows that the extracted leaves from the proposed method are more accurate compared to the segmentation-based method.

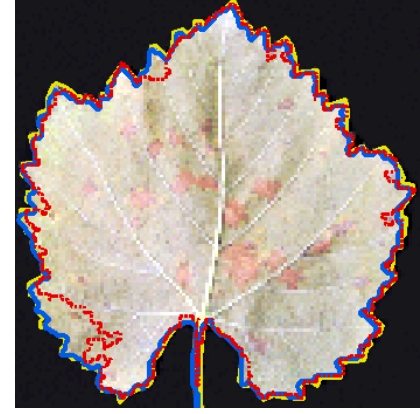

(a)

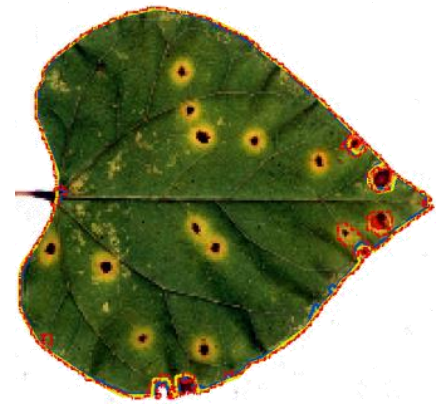

(b)
- Ground truth - Proposed method - Segmntation method

Figure 7. Extracted boundaries by different methods superimposed on the images in Figure 1.

The results in Table 1 indicate the enhanced capabilities of the proposed method to accurately extract leaf objects from images.

Table 1. Performance evaluation of the proposed method based the shape accuracy index

\begin{tabular}{|c|c|c|}
\hline Dataset & Proposed method & Segmentation-based \\
\hline $\begin{array}{c}\text { Image 1 } \\
\text { from Fig } \\
\text { 1(a) }\end{array}$ & $97 \%$ & $92 \%$ \\
\hline $\begin{array}{c}\text { Image 2 } \\
\text { from Figure } \\
\text { 1(b) }\end{array}$ & & \\
\hline
\end{tabular}

\section{CONCLUSION}

As plant diseases alter the geometry of leaves, it is important for a classification algorithm to have the ability to support a wide range of leaf shapes. This paper proposed a new geometry-led method that enables a classifier to implement a unified and dynamic geometry to model leaf objects. To construct a unified geometry, the method utilises a set of rules formulated based on a wingededge data structure. To enable a dynamic geometry for this structure, a collection of methods are applied through an iterative mechanism. We tested the proposed structure to model two different types of leaf objects and the results show the high performance of the proposed method in overcoming the limitations of conventional classification methods, such as using user-defined parameters and static geometry in modelling leaf spots. The implementation of a more complex scenario, e.g. a collection of leaves, and the integration of other spatial factors, would be interesting for further research.

\section{REFERENCES}

[1] Savary S., Ficke A., Aubertot J.N., Hollier C., "Crop Losses due to Diseases and their Implications for Global Food Production Losses and Food Security," Food Security, vol. 4, 2012, pp. 519-537.

[2] Singh V., Misra A.K, "Detection of plant leaf diseases using image segmentation and soft computing techniques". Information Processing in Agriculture vol.4, 2017, pp 41-49.

[3] Liang W-Z., Kirk K.R., Greene J. K., "Estimation of soybean leaf area, edge, and defoliation using color image analysis," Computers and Electronics in Agriculture, Vol. 150, 2018, pp. 41-51

[4] Sharif M., Khan M. Iqbal A, Z., Azam M.F., M.I.U. Lali, M.Y. Javed, "Detection and classification of citrus diseases in agriculture based on optimized weighted segmentation and feature selection", Comput. Electron. Agric. 150, 2018, 220234.

[5] Abu Bakar M.N., Abdullah A.H., Abdul Rahim N., Yazid H., Misman S.N. and Masnan M.J., "Rice Leaf Blast Disease Detection using Multi-Level Color Image Thresholding," Telecom, elec \& comp eng. vol 10, 1-15, 2018.

[6] Khan M. A., Lali M.I., Sharif M., Javed K., "An Optimized Method for Segmentation and Classification of Apple Diseases Based on Strong Correlation and Genetic Algorithm Based Feature Selection," in IEEE Access, vol. 7, pp. 4626146277, 2019,

[7] Jayawardena R.S., Liu M., Maharachchikumbura S., Zhang W., Xing Q., Hyde K., S. Nilthong, Liu X., Yan J., "Neopestalotiopsis vitis sp. Nov. causing grapevine leaf spot in China," Phytotaxa. 25, 2016, pp 63-74.

[8] Florida Division of Plant Industry, Florida Department of Agriculture and Consumer Services, Bugwood.org

[9] Hammam Y., A. Moore, \& P. Whigham, "The dynamic geometry of geographical vector Agents," Computers, Environment and Urban Systems 31(5), 2007, pp. 502-519.

[10] Moore A., "Geographical vector agent based simulation for agricultural land use modelling," Advanced Geo-Simulation Models, Bentham Science Publisher, 2011.

[11] Baumgart B. G., "A polyhedron representation for computer vision," In Proceedings of the May 19-22, 1975, national computer conference and exposition (AFIPS '75), ACM, New York, NY, USA, 589-596.

[12] Borna K., Moore A., and Sirguey P., "Towards a vector agent modelling approach for remote sensing image classification," Journal of Spatial Science, 59(2), 2014, 283-296.

[13] Borna K., 2017, "Geographic Vector Agents from Pixels to Intelligent Processing Units," (Thesis, Doctor of Philosophy). University of Otago. Retrieved from http://hdl.handle.net/10523/7183. 


\section{Authors' background}

\begin{tabular}{|l|l|l|l|}
\hline Your Name & Title $^{\star}$ & Research Field & Personal website \\
\hline Duaa Alshadli & lecturer & $\begin{array}{l}\text { Land Surveying- } \\
\text { photogrammetry-Image } \\
\text { classification }\end{array}$ & \\
\hline Kambiz Borna & lecturer & $\begin{array}{l}\text { GeoComputation- Remote } \\
\text { Sensing-Land Surveying }\end{array}$ & \\
\hline Cesar Lador & lecturer & Water management-GIS & \\
\hline
\end{tabular}

